\title{
Protocolo de atendimento odontológico a pacientes usuários de terapia antitrombótica
}

\section{Dental care protocol for patients using antithrombotic therapy}

\author{
Maura Sodré Pesse* \\ Leandro Dorigan de Macedo ${ }^{* *}$ \\ Soraya Fernandes Mestriner ${ }^{* * *}$ \\ Cristiane Aparecida Nogueira Bataglion ${ }^{* * *}$
}

\section{Resumo}

Muitos pacientes que necessitam de intervenção odontológica, atualmente, são usuários de terapia medicamentosa antitrombótica. Essa condição se mostra prevalente, considerando que problemas cardiovasculares afetam grande parte da população. Objetivo: construir um protocolo clínico para o Serviço de Odontologia e Estomatologia do Hospital das Clínicas de Ribeirão Preto da Faculdade de Medicina de Ribeirão Preto da Universidade de São Paulo, a partir de evidências científicas. Revisão de literatura: foi realizada uma pesquisa bibliográfica nas bases de dados eletrônicas Scientific Eletronic Library Online (SciELO) e Public MedLine (PubMed), nos idiomas português e inglês, do período entre 2013 e 2017. Após obtenção dos periódicos, foram selecionados os que se tratavam de estudos clínicos e seguiu-se com tradução, leitura e análise dos artigos. Com base nas evidências científicas, optou-se por construir o protocolo a partir da classe de medicamento utilizada pelos usuários de terapia antitrombótica (antiagregantes plaquetários, anticoagulantes orais e novos anticoagulantes orais) e orientações (pré, intra e pós-operatórias) para cada procedimento odontológico. Considerações finais: o protocolo clínico estabeleceu parâmetros para condutas clínicas e cirúrgicas em atendimento ambulatorial e hospitalar, possibilitando o cuidado integral aos usuários de terapia antitrombótica. Futuros estudos clínicos são necessários para validação e adequação para seu uso em diferentes serviços.

Palavras-chave: Agregação de plaquetas. Anticoagulantes. Fibrinolíticos. Inibidores. Odontologia. Protocolos clínicos.

\section{Introdução}

O tromboembolismo é um evento multifatorial e o risco é composto por fatores genéticos e/ou adquiridos. O principal fator de risco para trombose arterial é a arteriosclerose. A trombose venosa acontece em decorrência de imobilização, cirurgia, medicamentos, obesidade e condições genéticas. $\mathrm{O}$ evento tromboembólico resulta de um desequilíbrio entre o estímulo trombogênico e os mecanismos de proteção $0^{1,2}$.

A fim de prevenir, primária ou secundariamente, eventos tromboembólicos, são utilizadas terapias com anticoagulantes e/ou antiagregantes plaquetários. A terapia antitrombótica está indicada na prevenção de trombose venosa profunda, embolia pulmonar, trombose e embolização em pacientes com fibrilação atrial e trombose de válvulas cardíacas. É aplicada também em pacientes com prótese cardíaca, para síndromes coronarianas agudas, para pacientes que fazem uso de stents coronarianas, pós-acidentes vasculares isquêmicos cerebrais e cardíacos ${ }^{3-5}$.

Os antiagregantes plaquetários, além de apresentarem a propriedade de inibir a formação do trombo induzido predominantemente por plaquetas sem interferir de forma significativa nos demais segmentos da coagulação, são usados devido a evidências experimentais em que a adesão e agregação plaquetária ao endotélio vascular é o evento primário no desenvolvimento de trombose, tromboembolismo arterial, vasoespasmo coronariano e, possivelmente, no desenvolvimento de lesões ateroscleróticas. Os antiagregantes plaquetários mais comumente utilizados são ácido acetilsalicílico (AAS®), clopidogrel e ticlopidina ${ }^{6,7}$.

Residente do Programa de Atenção Integral à Saúde da Faculdade de Medicina de Ribeirão Preto da Universidade de São Paulo.

Chefe da Divisão de Odontologia e Estomatologia do Hospital das Clínicas da Faculdade de Medicina de Ribeirão Preto da Universidade de São Paulo.

Docente da Faculdade de Odontologia de Ribeirão Preto da Universidade de São Paulo.

Cirurgiã-dentista contratada do Hospital das Clínicas da Faculdade de Medicina de Ribeirão Preto da Universidade de São Paulo. 
Os anticoagulantes atuam diretamente na cascata de coagulação, tendo como objetivo prevenir a formação e a expansão do coágulo. As terapias incluem heparina, heparina de baixo peso molecular e anticoagulantes orais, sendo a varfarina o principal medicamento desse grupo ${ }^{8-11}$.

Recentemente, tornaram-se disponíveis no mercado duas novas classes de anticoagulantes orais: os inibidores do fator Xa (rivaroxabana, apixabana e edoxabana) e os inibidores diretos da trombina (dabigatrana). Esses medicamentos surgiram como alternativas aos anticoagulantes cumarínicos e parenterais, apresentam perfil IV farmacológico previsível, são administrados oralmente e não necessitam de monitorização laboratorial frequente ${ }^{12}$.

Há uma grande preocupação com pacientes sob terapia anticoagulante em relação ao risco de complicações hemorrágicas ou de ocorrência de eventos tromboembólicos, principalmente em relação aos anticoagulantes antagonistas da vitamina $\mathrm{K}$ (ex.: varfarina), que sofrem influência na absorção, farmacocinética e farmacodinâmica em função de fatores genéticos, ambientais e pela ingestão de alguns tipos de alimentos ${ }^{13-17}$.

As complicações hemorrágicas em função de procedimentos odontológicos que necessitem de mais do que medidas hemostáticas locais para esses pacientes são raras $(0,1 \%)$, sendo que não há nenhum relato de hemorragias fatais. Por outro lado, 0,8\% apresentam complicações tromboembólicas decorrente da suspensão da terapia antitrombótica e, dentre esses, há relatos de desfechos fatais. Considerando que muitos pacientes que precisam de intervenção odontológica são submetidos a terapias antitrombóticas e sendo essa situação cada vez mais expressiva, é necessário que se crie um protocolo de atendimento que minimize as ocorrências hemorrágicas e não exponha o paciente a um risco de tromboembolismo. Protocolos são considerados importantes instrumentos para direcionamento das situações na assistência em saúde e, nesse caso, apresentam como foco principal a padronização de condutas clínicas em ambientes ambulatoriais e hospitalares ${ }^{18-20}$.

Assim, com base nesses preceitos, a finalidade deste estudo foi construir um protocolo clínico destinado ao atendimento odontológico para usuários de terapia antitrombótica, baseando-se na literatura existente sobre o tema.

\section{Materiais e método}

Foi realizada revisão bibliográfica exploratória nas bases de dados eletrônicas: Scientific Eletronic Library Online (SciELO) e Public MedLine (PubMed), com obtenção de periódicos, seguida de seleção, tradução, leitura e análise de artigos. A pesquisa dos periódicos aconteceu entre julho e dezembro de 2017. Os descritores em inglês e português utilizados foram: "assistência odontológica/dental care", "anticoagulantes/anticoagulants", "extração dentária/tooth extraction" e "fibrinolíticos/fibrinolytic agents"; selecionados dos Descritores em Ciência da Saúde da Biblioteca Virtual em Saúde (BVS) edição 2017. Foram encontrados 44 artigos científicos, dos quais foram selecionados 19 estudos clínicos nos idiomas português e inglês, publicados no período entre 2013 e 2017.

A partir da leitura e da análise dos artigos, com base nas evidências científicas, optou-se por construir o protocolo clínico a partir da classe de medicamento utilizada pelos usuários de terapia antitrombótica, com antiagregantes plaquetários (terapia única ou dupla), anticoagulantes orais, anticoagulantes orais associados a antiagregantes plaquetários e novos anticoagulantes orais; orientações pré-operatórias, solicitação de exames, parâmetros de Razão Normalizada Internacional (International Normalized Ratio - INR), suspensão ou manutenção da medicação; orientações intraoperatórias, para determinar extensão e complexidade do procedimento e uso de hemostáticos locais; orientações pós-operatórias, cuidados pós-operatórios; para cada tipo de procedimento odontológico, restauração, raspagem supragengival, raspagem subgengival, biopulpectomia/pulpotomia, necropulpectomia, biópsia, extração simples, extração complexa (quando envolve osteotomia e/ou retalho) e extração de elementos inclusos.

O protocolo foi organizado de forma que, para cada classe de medicamento, foram descritas as orientações de acordo com o período pré, intra e pós-operatório, conforme o tipo de procedimento odontológico. 


\section{Resultados}

Quadro 1 - Protocolo clínico

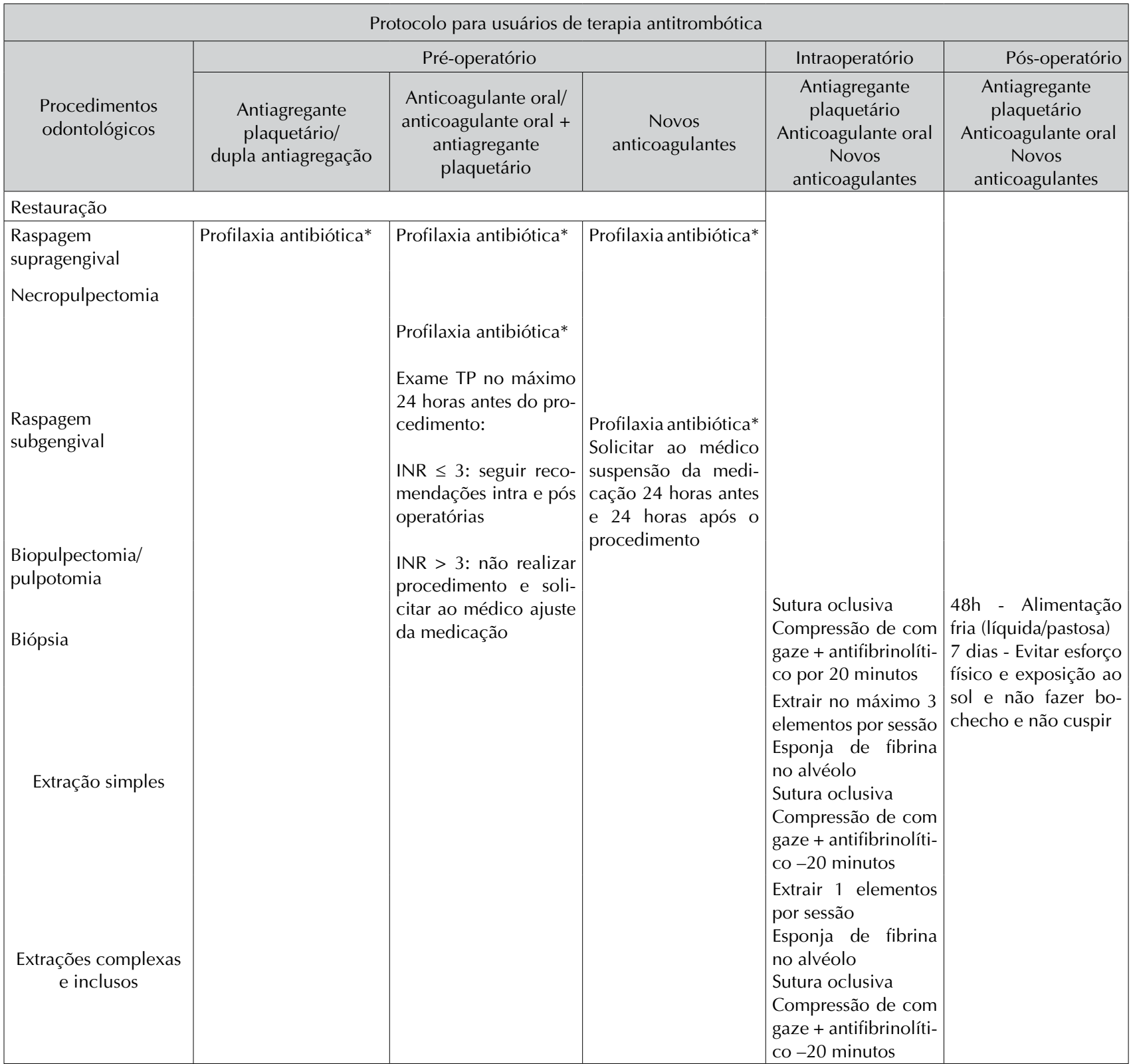

* Realizar a profilaxia antibiótica para os casos em que há risco de endocardite infecciosa, segundo critérios de American Heart Association (AHA). Fonte: autores.

Quadro 2 - Considerações protocolo clínico

* Condições cardíacas consideradas de alto risco para a endocardite infecciosa, nas quais a profilaxia antibiótica é recomendada previamente aos procedimentos odontológicos

- Valvas cardíacas protéticas ou material protético usado para reparo de válvulas cardíacas;

- História prévia de endocardite infecciosa;

- Doenças cardíacas congênitas (DCC):

- DCC cianótica não reparada, incluindo casos com shunts e condutos paliativos;

- defeito cardíaco congênito completamente reparado com material ou dispositivo protético, se colocado por cirurgia ou intervenção com cateteres, durante os primeiros 6 meses após o procedimento;

- DCC reparada com defeitos residuais no sítio ou adjacente a ele de um curativo ou dispositivo protético (inibem a endotelização)

- Pacientes que receberam transplante cardíaco e desenvolveram valvulopatia cardíaca.

\section{Terapêutica}

A profilaxia antibiótica deve ser realizada com $2 \mathrm{~g}$ de amoxicilina 1 hora antes do procedimento ou, para alérgicos à penicilina, 600 mg de clindamicina 1 hora antes do procedimento.

Hemostasia local

Deve-se realizar introdução de esponja de fibrina no interior do alvéolo, sutura oclusiva e compressão com gaze embebida com 5 mL de ácido aminocaproico $200 \mathrm{mg} / \mathrm{mL}$ ou com $5 \mathrm{ml}$ de ácido tranexâmico $50 \mathrm{mg} / \mathrm{mL}$ durante 20 minutos.

Anestésico local

Utilizar prilocaína 3\% felipressina 0,03UI ou, no máximo, 2 tubetes de lidocaína 2\% epinefrina 1:100 000.

Fonte: autores. 


\section{Discussão}

Os protocolos são recomendações desenvolvidas sistematicamente para auxiliar no manejo de um problema de saúde, numa circunstância clínica específica, preferencialmente baseados na melhor informação científica. São orientações concisas sobre testes diagnósticos e tratamentos que podem ser facilitadores no dia a dia. Além disso, são importantes ferramentas para atualização na área da saúde, sendo utilizados para reduzir variações inapropriadas na prática clínica ${ }^{21}$.

No caso dos usuários de terapia antitrombótica e que necessitam de tratamento odontológico, faz-se necessário um planejamento adequado, que inclua avaliação completa da condição sistêmica do paciente, monitoramento do nível de coagulação por exames laboratoriais, classificação do trauma e da extensão do procedimento a ser realizado; e que estabeleça um direcionamento sobre a conduta em relação ao medicamento utilizado e hemostático lo$\mathrm{cal}^{18}$.

A literatura propõe que, a cada 1.000 pacientes, 8 apresentam complicações embólicas graves quando a anticoagulação é reduzida ou retirada para procedimentos dentários, sendo que para 2 desses pacientes os eventos são fatais ${ }^{20,22}$.

Assim, consideramos que a manutenção da medicação é a melhor opção quando se tratam das complicações embólicas que o paciente pode apresentar quando ocorre a suspensão da medicação. No entanto, precisamos estabelecer parâmetros seguros aos pacientes expostos a um risco mínimo de sangramento, principalmente aquele que exige intervenção sistêmica.

As diretrizes europeias não recomendam que exames de agregação plaquetária sejam feitos de forma rotineira para usuários de antiagregantes plaquetários, mas o teste pode ser feito para casos específicos, particularmente para usuários de clopidogrel, devido à sua alta taxa de resistência terapêutica. Para os demais casos, não se torna necessário, porque as taxas de antiagregação mantêm-se estáveis após 30 dias de uso da medicação e são principalmente dependentes da dose utilizada sem sofrer influências externas ${ }^{23}$.

O exame de escolha para monitorização dos anticoagulantes cumarínicos (sendo a varfarina o principal medicamento dessa classe) é o tempo de protrombina (TP), já que, dos cinco fatores que influenciam o TP, três são dependentes da vitamina K (II, VII, X). Para que ocorra padronização e o TP possa ser comparável em qualquer laboratório do mundo, seu valor é expresso em INR, critério proposto pela Organização Mundial de Saúde (OMS) desde $1982^{24-26}$. O valor de INR para indivíduos normais é de aproximadamente 1 . $O$ valor terapêutico do INR indicado para a maioria das condições tromboembólicas deve estar entre 2,0 e 3,0; exceto em situações particulares, em que se recomenda INR entre 2,5 e $3,5^{27-29}$.

Existem controvérsias em relação ao valor máximo de INR para se realizar procedimentos cirúrgicos odontológicos. Alguns autores, incluindo a Sociedade Japonesa de Cirurgiões Orais, consideram seguro valor de INR até $3,0^{30-32}$. Enquanto outros, incluindo recomendações do Comitê de Saúde Britânico e da Sociedade Brasileira de Cardiologia, indicam valor máximo de INR até 4,0 , desde que se utilizem medidas hemostáticas locais ${ }^{33-35}$.

Daroit et al. ${ }^{35}$ (2017) concluíram que pequenas cirurgias orais podem ser realizadas de forma segura em paciente em uso de anticoagulante oral desde que se utilize INR abaixo de 4,0, tenha-se um bom planejamento do caso, utilizando técnicas atraumáticas, extraindo um elemento dentário por sessão e utilizando hemostáticos locais. Al-Mubarak et al. ${ }^{36}$ (2007) e Morimoto et al. ${ }^{37}$ (2008) não encontraram diferença estatisticamente significante no sangramento pós-operatório em seus respectivos casos para um valor máximo de 3,0 de INR. Bacci et al. ${ }^{33}$ (2010) relataram como seguro INR de até 4,0. Já Ferrieri et al. ${ }^{38}$ (2007) não encontraram diferença estatística em relação ao sangramento pós-operatório, entre controles e casos, com um INR não superior a 5,5 .

De acordo com as recomendações internacionais atuais os pacientes com INR superior a 4,0 apresentam um aumento considerável do risco de sangramento sem benefício clínico ${ }^{33,39,40}$. Sendo que pacientes com INR entre 2,0 e 3,0 apresentam risco de sangramento aumentado, de aproximadamente 1 em 100 a 1 em 25. Para aqueles com INR superior a 3,0 , o risco de sangramento aumenta para aproximadamente $1 \mathrm{em} 30$ a $1 \mathrm{em} 7^{41}$.

No protocolo proposto neste estudo, estabelecemos valor de INR máximo de 3,0, já que, segundo Todd $^{42}$ (2005), de maneira geral, o INR alvo para controle tromboembólico para a maioria das condições clínicas está entre 2,0 e 3,0. Além disso, alguns estudos encontraram relação entre um aumento do INR e a incidência de hemorragia no pós-operatório após o procedimento ${ }^{36,43-45}$.

Febbo et al. ${ }^{41}$ (2016) encontraram relação estatisticamente significativa entre maior valor de INR, número de dentes extraídos e risco de sangramento pós-operatório. Ainda, relataram que, dos 9 casos de sangramento pós-operatório, que correspondeu a $2,1 \%$ da amostra, 3 casos foram relacionados a extrações simples e 6 casos a extrações múltiplas ${ }^{34}$.

Hanken et al. ${ }^{46}$ (2016) recomendam que procedimentos múltiplos sejam divididos em várias sessões, a fim de reduzir o risco de sangramento, lembrando que a complexidade do procedimento é também determinada pelo fato de envolver osteotomia e/ou retalho. A incidência de hemorragia pós-operatória foi maior após extrações dentárias que envolviam retalho e/ou osteotomia ${ }^{32}$. 
A European Heart Rhythm Association (EHRA) considera como intervenções de baixo risco de sangramento extrações de até três dentes, sendo que extrações envolvendo osteotomia aumentam o trauma cirúrgico. Assim, é interessante que se façam extrações de até três elementos tratando-se de extrações simples, já quando envolverem osteotomia ou retalho, esse número deve ser reduzido. $\mathrm{O}$ que é confirmado pelos resultados obtidos por Dudek et al. ${ }^{47}$ (2016), que encontraram associação estatística entre risco hemorrágico e quantidade de dentes extraídos, e entre risco hemorrágico e osteotomia.

$\mathrm{O}$ uso de medidas locais de hemostasia esteve presente em todos os trabalhos analisados. Alguns utilizaram apenas os métodos usuais, como sutura e compressão com gaze; outros se valeram de medidas adicionais, como esponja de fibrina, irrigação do alvéolo com agente antifibrinolítico, bochechos com antifibrinolíticos, compressão com gaze embebida em antifibrinolíticos e cola de fibrina. Medidas hemostáticas locais combinadas resultaram em menor frequência de sangramento pós-operatório $(6,8 \%)$, com uso de esponja de fibrina no interior do alvéolo, sutura e compressão de gaze embebida com ácido tranexâmico ${ }^{47}$.

Em uma amostra de 439 pacientes, em que 9 retornaram com sangramento pós-operatório, todos, exceto um caso, foram resolvidos com medidas locais de hemostasia. Foram realizadas a irrigação do alvéolo com ácido tranexâmico, a colocação de esponja de fibrina no alvéolo e a compressão com gaze embebida com ácido tranexâmico. Além disso, os pacientes foram orientados a manter enxágue bucal com ácido tranexâmico por dois dias. Somente um caso exigiu internação hospitalar seguida de transfusão sanguínea, sendo que, no momento da internação, foi identificado INR superior a $5,0^{34}$.

As recomendações como evitar exposição ao sol e realização de esforços físicos por sete dias visam à prevenção da hemorragia por evitar vasodilatação. As orientações em relação à alimentação líquida/ pastosa fria por pelo menos 48 horas promovem um repouso da área e, consequentemente, reduzem o risco hemorrágico ${ }^{48}$.

Com relação ao tipo de terapia antitrombótica, em um estudo com 270 pacientes submetidos à extração dentária, sendo 134 em terapia com varfarina, 49 com associação entre varfarina e antiagregantes plaquetários e $87 \mathrm{com}$ antiagregantes plaquetários, 11 pacientes apresentaram complicações hemorrágicas. Dos 11 casos, 7 utilizavam a varfarina isolada, 2 combinavam varfarina e antiagragante plaquetáro e 2 utilizavam somente antiagregante plaquetário. Não houve relação estatisticamente significativa entre os grupos; o sangramento pós-operatório e todas as complicações foram resolvidos com medidas locais de hemostasia ${ }^{37}$.

Outro estudo não apresentou diferença estatística entre pacientes submetidos a extrações dentárias e que usavam terapia antiplaquetária simples ou dupla antiagregação. Em todos os casos, o uso de hemostáticos locais foi suficiente para garantir a hemostasia, e não ocorreu nenhum episódio prolongado de sangramento pós-operatório ${ }^{5}$. Quando comparados grupos em que se manteve a antiagregação e grupo em que a medicação foi suspensa e grupo controle, a hemostasia foi satisfatória para todos os 82 pacientes da amostra, a hemostasia foi satisfatória sem episódios de sangramento ${ }^{49}$.

Para os novos anticoagulantes (dabigratana, rivaroxabana e apixabana), de uma forma geral, é recomendada a suspensão 24 horas antes do procedimento, com retorno 24 horas após. Já que, mesmo na ausência de estudos controlados, esse intervalo, que é baseado na meia-vida plasmática desses medicamentos, garante segurança hemostática e uma menor exposição ao evento tromboembólico, uma vez que o tempo sem a cobertura da medicação é reduzido para 24-48 horas, quando comparados com os anticoagulantes convencionais, que exigem, em média, de 6 a 7 dias ${ }^{50}$.

Morimoto et al. ${ }^{51}$ (2016) propuseram um estudo em que realizaram extrações dentárias sem descontinuar a terapia com novos anticoagulantes. De acordo com os achados do estudo, os autores recomendam que a extração deve ser evitada nas 3 horas seguintes à ingestão da medicação, que é quando ocorre o pico plasmático (as extrações foram feitas 6 horas após a ingestão do medicamento). Acrescenta-se que devem ser realizados acompanhamento pós-operatório e utilização de hemostáticos locais. Se a hemostasia não estiver adequada, deve-se suspender a próxima dose da medicação, após discussão com médico. Essas considerações não se aplicam para pacientes com disfunção renal ou hepática.

Miranda et al. ${ }^{50}$ (2016) suspenderam os novos anticoagulantes de 12 a 24 horas antes do procedimento, reintroduzindo a medicação no dia seguinte ao procedimento. Após exodontia, utilizaram medidas hemostáticas adicionais, com irrigação do alvéolo com ácido tranexâmico, colocação de esponja de fibrina intra-alveolar e compressão com gaze embebida com ácido tranexâmico durante 15 minutos. O sangramento foi avaliado imediatamente após a extração, após 24 horas, após 72 horas e após 7 dias. Não foram observados sangramentos intra ou pós-operatórios.

Pensando na redução das complicações, não recomendamos que seja realizado procedimento cirúrgico sem a suspensão da medicação, no caso dos anticoagulantes orais novos, já que, exceto em um estudo, todos os outros revisados indicaram que sejam realizadas a suspensão da medicação e a utilização de medidas hemostáticas locais.

Apesar de todas as implicações envolvidas e de os protocolos serem instrumentos úteis na organização do processo de trabalho e rosolutividade das ações, é importante lembrar que o emprego de protocolos apresenta limites, por se tratarem de procedimentos preestabelecidos, sem considerar que a 
clínica apresenta significativa variedade de demanda. Sendo assim, deve-se combinar a padronização dos procedimentos, sem deixar de lado as variações presentes em cada caso ${ }^{19,52}$.

\section{Considerações finais}

Com base na pesquisa bibliográfica, concluímos que os procedimentos de restauração, raspagem supragengival e necropulpectomia podem ser realizados sem recomendações específicas, além da profilaxia antibiótica para os casos recomendados, de acordo com a AHA. Para os procedimentos invasivos, independente da medicação utilizada, é necessário seguir os parâmetros recomendados em relação a exames, extensão e trauma do procedimento. Para todos os casos, devem ser utilizadas medidas locais de hemostasia.

O protocolo apresentado funciona como norteador para que ocorra o cuidado integral do paciente usuário de terapia antitrombótica que necessita de tratamento odontológico. No entanto, são necessários estudos clínicos futuros para validação desse protocolo.

\section{Abstract}

Several patients who require dental intervention are current users of antithrombotic drug therapy. This condition is prevalent, considering that cardiovascular problems affect a large portion of the population. Objective: this study aimed to construct a clinical protocol for the Service of Dentistry and Stomatology of the Clinics Hospital of Ribeirão Preto from the Medical School of Ribeirão Preto of the University of São Paulo, Brazil, based on scientific evidence. Literature review: a bibliographic review was performed in the Scientific Electronic Library Online (SciELO) and Public MedLine (PubMed) electronic databases, in the Portuguese and English languages, for the period of 2013 through 2017. After obtaining the journals, the clinical studies were selected, followed by the translation, reading, and analysis of the articles. Based on the scientific evidence, we decided to build the protocol from the class of medication of the users of antithrombotic therapy (antiplatelet agents, oral anticoagulants, and new oral anticoagulants), and guidelines (pre-, intra-, and postoperative) for each dental procedure. Final considerations: the clinical protocol established parameters for clinical and surgical procedures in outpatient and hospital care, allowing comprehensive care to users of antithrombotic therapy. Further clinical studies are required to validate and adequate the use of this therapy in different services.

Keywords: Aggregation of platelets. Anticoagulants. Fibrinolytics. Inhibitors. Dentistry. Clinical protocols.

\section{Referências}

1. Llanos, B. Nuevas perspectives en el tratamiento antitrombótico. Información Terapéutica del Sistema Nacional de Salud 2001; 25(4):93-104.

2. Forchesatto Filho L, Barros E. Coagulação: trombose arterial e venosa. Medicina interna na prática clínica. Porto Alegre: Artmed; 2013. p. 99-406.

3. Schulman S. Care of patients receiving long-term anticoagulant therapy. N Engl J Med 2003; 349:675-83.

4. Pototski M, Amenábar JM. Dental management of patients receiving anticoagulation or antiplatelet treatment. J Oral Sci 2007; 49(4):253-8.

5. Napeñas JJ, Hong CH, Brennan MT, Furney SL, Fox PC, Lockhart PB. The frequency of bleeding complications after invasive dental treatment in patients receiving single and dual antiplatelet therapy. J Am Dent Assoc 2009; 140(6):690-5.

6. Silva, P. Farmacologia. 8. ed. Rio de Janeiro: Guanabara Koogan; 2010. p. 593-4.

7. Roblers P, Michailidis DP, Stansby G. Systematic review of antiplatelet therapy for the prevention of myocardial infarction, stroke or vascular death in patients with peripheral vascular disease. Brit J Surgery 2001; (88):787-800.

8. Rodriguez-Cabrera MA, Barona-Dorado C, Leco-Berrocal I, Gomez-Moreno G, Martinez-González JM. Extracions Without eliminating anticoagulant treatment: a literature review. Med Oral Patol Oral Cir Bucal 2011; 1:16(6):800-4.

9. Majerus PW, Tollefsen DM. Anticoagulantes, trombolíticos e fármacos antiplaquetários. In: Goodman LS, Gilman A. As bases farmacológicas da terapêutica. . ed. Rio de Janeiro: McGraw Hill; 2005. p. 1141-55.

10. Silva P. Farmacologia. 6. ed. Rio de Janeiro: Guanabara Koogan; 2002.

11. Rang HP, Dale MM, Ritter JM, Flower RJ, Henderson G. Farmacologia. 5. ed. Rio de Janeiro: Elsevier; 2003.

12. Fernandes CJCS, Alves JL Jr, Gavilanes F, Prada LF, Morinaga LK, Souza R. Os novos anticoagulantes no tratamento do tromboembolismo venoso. J Bras Pneumol 2016; 42(2):146-54.

13. Santos FC, Meffei FHA, Carvalho LR, Tomazini-Santos IA, Gianini M, Sobreira ML, et al. Complicações da terapia anticoagulante com warfarina em pacientes com doença vascular periférica: estudo coorte prospectivo. J Vascular Bras 2006; 5(3):194-202

14. Lourenço DM, Lopes LHC, Vignal CV, Morelli VM. Avaliação clínica e laboratorial de pacientes em uso de anticoagulantes orais. Arq Bras Cardiologia 1997; 68(5):353-6.

15. Silva KR, Costa R, Abi-Rached R, Filho MM, Caldas JGMP, Carnevale FC, et al. Warfarin prevents venous obstruction after cardiac devices implantation in high-risk patients: partial analysis. Rev Bras Cir Cardiovascular 2008; 23(4):542-9.

16. Biederman FA, Carvalho ARS, Piccoli M. Uso de anticoagulantes orais: atenção especial com a nutrição. $2 \square$ Seminário Nacional Estado e Políticas Sociais no Brasil; 2005; Cascavel: Unioeste Campus de Cascavel; 2005.

17. Molina FT, Zanusso GJ. Anticoagulantes Cumarínicos: ações, riscos e monitoramento da terapêutica. SaBios: Rev Saúde e Biol 2014; 9(2):75-82.

18. Dantas AK, Deboni MC, Piratininga JL. Cirurgias odontológicas em usuários de anticoaguantes orais. Rev Bras Hematol Hemoter 2009; 31(5):337-40.

19. Werneck MAF, Faria HP, Campos KFC. Protocolo de cuidados à saúde e de organização do serviço. Belo Horizonte: Coopmed; 2009. p. 10-2.

20. Wahl MJ, Pinto A, Kilham J, Lalla RV. Dental surgery in anticoagulated patients - stop the interruption. Oral Surgery Oral Med Oral Pathol Oral Radiology 2015; 119:136-57. 
21. BRASIL. Ministério da Saúde. Grupo Hospitalar Conceição/ Gerência de Ensino e Pesquisa. Diretrizes Clínicas/Protocolo Assistenciais. Manual Operacional. Porto Alegre: 2008. p. 11.

22. Clemm R, Neukam FW, Rusche B, Bauersachs A, Musazada $\mathrm{S}$, Schmitt CM. Manegment of Anticoagulanted therapy in implant dentistry: a clinical comparative study. Clin Oral Impl Res 2016; 27(10)1274-82.

23. Bertrand ME, Chair ML, Simoons KA, Fox A, Wallentin LC, Hamm CW, et al. ESC Guidelines for management of acute coronary syndromes in patients presenting without persistent ST-segment elevation of European Society cardiology (ESC). Eur Heart J 2011; 32(23):2999-3054.

24. Clé DV, Garcia AA, Brunetta DM, Schwartzmann PV, Moriguti J. Anticoagulação em pacientes hospitalizados In: Simpósio Condutas em enfermaria de clínica médica de hospital de média complexidade, 2010; Ribeirão Preto: Medicina; 43(2):107-17.

25. Lavítola PL, Spina GS, Sampaio RO, Tarasoutchi F, Ginberg M. Sangramento durante a anticoagulação oral: alerta sobre um mal maior. Arq Bras Cardiologia 2009; 93(2):174-9.

26. Franco RF. Fisiologia da coagulação, anticoagulação e fibrinólise. In: Simpósio de medicina hemostasia e trombose, 2011; Ribeirão Preto: 34. p. 229-37.

27. Muthukrishnan A, Bishop K. An assessment of the management of patients on warfarin by general dental practitioners in South West Wales [resumo]. Br Dent J 2003; 22:195(10):567.

28. Wannmacher L. Antiagregantes plaquetários, anticoagulantes e hemostáticos. In: Wannmacher L, Ferreira MBC. Farmacologia clínica para dentistas. 3. ed. Rio de Janeiro: Guanabara Koogan; 2007. p. 395-9.

29. Ansell J, Hirsh J, Hylek E, Jacobson A, Crowther M, Palareti G. Pharmacology and management of the vitamin K antagonists: American College of Chest Physicians Evidence-Based Clinical Practice Guidelines (8th Edition). Chest 2008; 133(6):160-98

30. Iwabuchi H, Imai Y, Asanami S, Shirakawa M, Yamane GY, Ogiuchi H, et al. Evaluation of postextraction bleeding incidence to compare patients receiving and not receiving warfarin therapy: a cross-sectional, multicentre, observational study. BMJ Open 2014; 4:e005777.

31. Dudek D, Marchionni S, Gabriele M, Iurlaro A, Helewski K, Toti $\mathrm{P}$, et al. Bleeding rate after tooth extraction in patients under oral anticoagulant therapy. J Craniofac Surg 2016; 27:1228-33.

32. Ohba S, Yoshimura H, Matsuda S, Kobayashi J, Kimura T, Aiki M, et al. Risk factors for postoperative hemorrhage after minor oral surgery in patients treated with antithrombotic agents. Odontology 2015; 103(2):227-32.

33. Bacci C, Maglione M, Favero L, Perini A, Di Lenarda R, Berengo $\mathrm{M}$, et al. Management of dental extraction in patients undergoing anticoagulant treatment. Results from a large, multicenter, prospective, case-control study. Thromb Haemost 2010; 104:972-5.

34. Febbo A, Cheng A, Stein B, Goss A, Sambrook P. Postoperative bleeding following dental extractions in patients anticoagulated with warfarin. J Oral Maxillofac Surg 2016; 74:1518-23.

35. Daroit NB, Festugatto FL, Beltrame LF, Maciel JCC, Rados PV. Analysis of profiles of patients on oral anticoagulants undergoing dental extraction - a retrospective study J Oral Diag 2017; 02:e20170018.

36. Al-Mubarak S, Al-Ali N, Rass M, Al-Sohail A, Robert A, Al-Zoman K, et al. Evaluation of dental extractions, suturing and INR on postoperative bleeding of patients maintained onoral anticoagulant therapy. Br Dent J 2007; 203(E15).

37. Morimoto Y, Niwa H, Minematsu K. Hemostatic management of tooth extractions in patients on oral antithrombotic therapy. J Oral Maxillofac Surg 2008; 66:51.
38. Ferrieri GB, Castiglioni S, Carmagnola D, Cargnel M, Strohmenger L, Abati S. Oral surgery in patients on anticoagulant treatment without therapy interruption. J Oral Maxillofac Surg 2007; 65:1149.

39. Weltman NJ, Al-Attar Y, Cheung J, Duncan DPB, Katchky A, Azarpazhooh A, Abrahamyan L. Managment of dental extraction in patients taking warfarin as anticoagulant treatment: systematic review. Can Dent Assoc 2015; 81:20.

40. Lockhart P, Gibson J, Pond SH, Leitch J. Dental manegment considerations for the patient with an acquired coagulophaty. Part 2: Cooagulapathies from drugs. Brit Dent J 2003; 195:405-501.

41. Febbo A, Cheng A, Stein B, Goss A, Sambrook P. Postoperative bleeding following dental extractions in patients anticoagulated with warfarin. J Oral Maxillofac Surg 2016; 74:1518-23.

42. Todd DW. Evidence to support an individualized approach to modification of oral anticoagulant therapy for ambulatory oral surgery. J Oral Maxillofac Surg 2005; 63:536-9.

43. Blinder D, Manor Y, Martinowitz U, Taicher S. Dental extractions in patients maintained on oral anticoagulant therapy: comparison of INR value with occurrence of postoperative bleeding. Int J Oral Maxillofac Surg 2001; 30(6):518-21.

44. Scully C, Wolff A. Oral surgery in patients on anticoagulant therapy. Oral Surg Oral Med Oral Pathol Oral Radiol Endod 2002; 94(1):57-64.

45. Bodner L, Weinstein JM, Baumgarten AK. Efficacy of fibrin sealant in patients on various levels of oral anticoagulant undergoing oral surgery. Oral Surg Oral Med Oral Pathol Oral Radiol Endod 1998; 86(4):421-4.

46. Hanken H, Grobe A, Heiland M, Smeets R, Kluwe L, Wikner $\mathrm{J}$, et al. Postoperative bleeding risk for oral surgery under continued rivaroxaban anticoagulant therapy. Clin Oral Invest 2016; 20(6):1279-82.

47. Dudek D, Marchionni S, Gabriele M, Iurlaro A, Helewski K, Toti $\mathrm{P}$, et al. Bleeding rate after tooth extraction in patients under oral anticoagulant therapy. J Craniofac Surg 2016; 27(5):1228-33.

48. Nogueira AS, Vasconcelos BC, Frota R, Cardoso AB. Orientações pós-operatórias em cirurgia bucal. J Bras Clin Odontol Int 2006; Edição Especial:01-06.

49. Krishnan B, Shenoy NA, Alexander M. Exodontia and antiplatelet therapy. J Oral Maxillofac Surg 2008; 66(10):2063

50. Miranda M, Martinez LS, Franco R, Forte V, Barlattani A, Bollero P. Differences between warfarin and new oral anticoagulants in dental clinical practice. Oral \& Implantology $2016 ; 9(3): 151-6$

51. Morimoto Y, Yokoe C, Imai Y, Sugihara M, Futatsuki T. Tooth extraction in patients taking nonvitamin $\mathrm{K}$ antagonist oral anticoagulants. J Dent Sciences 2016; 11:59-64.

52. Campos VS, Amaral MA. A clínica ampliada e compartilhada, a gestão democrática e redes de atenção como referências teórico operacionais para reforma de hospital. Ciênc e Saúde Colet $2007 ; 12(4): 849-59$

\section{Endereço para correspondência:}

Maura Sodré Pesse

Rua Dr. Mário de Assis Moura, 480, Apto 172 A,

Jardim Nova Aliança,

14026578-000, Ribeirão Preto, São Paulo, Brasil

Telefone: (16) 992033637

E-mail:maurapesse@uol.com.br

Recebido: 05/04/18. Aceito: $11 / 07 / 18$. 